\title{
New insights into intrinsic foot muscle morphology and composition using ultra- high-field (7-Tesla) magnetic resonance imaging
}

Melinda M. Franettovich Smith ${ }^{1 *}$, James M. Elliott ${ }^{1,2,3}$, Aiman Al-Najjar ${ }^{4}$, Kenneth A. Weber $\|^{5}$, Mark A. Hoggarth ${ }^{3}$, Bill Vicenzino ${ }^{1}$, Paul W. Hodges ${ }^{1}$ and Natalie J. Collins ${ }^{1,6}$

\begin{abstract}
Background: The intrinsic muscles of the foot are key contributors to foot function and are important to evaluate in lower limb disorders. Magnetic resonance imaging (MRI), provides a non-invasive option to measure muscle morphology and composition, which are primary determinants of muscle function. Ultra-high-field (7-T) magnetic resonance imaging provides sufficient signal to evaluate the morphology of the intrinsic foot muscles, and, when combined with chemical-shift sequences, measures of muscle composition can be obtained. Here we aim to provide a proof-of-concept method for measuring intrinsic foot muscle morphology and composition with highfield MRI.
\end{abstract}

Methods: One healthy female (age 39 years, mass $65 \mathrm{~kg}$, height $1.73 \mathrm{~m}$ ) underwent MRI. A T1-weighted VIBE radio-frequency spoiled 3D steady state GRE - sequence of the whole foot was acquired on a Siemens $7 T$ MAGN ETOM scanner, as well as a 3T MAGNETOM Prisma scanner for comparison. A high-resolution fat/water separation image was also acquired using a 3D 2-point DIXON sequence at 7T. Coronal plane images from 3T and 7T scanners were compared. Using 3D Slicer software, regions of interest were manually contoured for each muscle on 7T images. Muscle volumes and percentage of muscle fat infiltration were calculated (muscle fat infiltration \% = Fat/ (Fat + Water) x100) for each muscle.

Results: Compared to the $3 \mathrm{~T}$ images, the $7 \mathrm{~T}$ images provided superior resolution, particularly at the forefoot, to facilitate segmentation of individual muscles. Muscle volumes ranged from $1.5 \mathrm{~cm}^{3}$ and $19.8 \mathrm{~cm}^{3}$, and percentage muscle fat infiltration ranged from $9.2-15.0 \%$.

(Continued on next page)

\footnotetext{
* Correspondence: melinda.smith@uq.edu.au

${ }^{1}$ School of Health and Rehabilitation Sciences, The University of Queensland, 4072 Brisbane, QLD, Australia

Full list of author information is available at the end of the article
}

C C The Author(s). 2021 Open Access This article is licensed under a Creative Commons Attribution 4.0 International License, which permits use, sharing, adaptation, distribution and reproduction in any medium or format, as long as you give appropriate credit to the original author(s) and the source, provide a link to the Creative Commons licence, and indicate if changes were made. The images or other third party material in this article are included in the article's Creative Commons licence, unless indicated otherwise in a credit line to the material. If material is not included in the article's Creative Commons licence and your intended use is not permitted by statutory regulation or exceeds the permitted use, you will need to obtain permission directly from the copyright holder. To view a copy of this licence, visit http://creativecommons.org/licenses/by/4.0/ The Creative Commons Public Domain Dedication waiver (http://creativecommons.org/publicdomain/zero/1.0/) applies to the data made available in this article, unless otherwise stated in a credit line to the data. 
(Continued from previous page)

Conclusions: This proof-of-concept study demonstrates a feasible method of quantifying muscle morphology and composition for individual intrinsic foot muscles using advanced high-field MRI techniques. This method can be used in future studies to better understand intrinsic foot muscle morphology and composition in healthy individuals, as well as those with lower disorders.

Keywords: Magnetic resonance imaging, Foot, Muscle fat infiltration, Plantar intrinsic muscles, Foot core, Morphology, Musculoskeletal imaging, Muscle segmentation

\section{Background}

The intrinsic foot muscles are those that have their anatomical attachments within the foot, in contrast to the extrinsic muscles that originate on the leg and insert on the foot. Together with passive structures (such as the bony arches, fat pads, ligaments and plantar fascia), the intrinsic foot muscles assist attenuation of forces associated with the foot-ground collision and stiffening of the foot for propulsion $[1,2]$. The distinguishing contribution of the intrinsic foot muscles, compared to passive structures, is the ability to modulate the energetic function of the foot to respond to changing demands (e.g. acceleration and deceleration, surfaces and footwear) [1, 3].

The important contribution of the intrinsic foot muscles to foot function suggests they should be considered when evaluating and treating patients with lower limb disorders. The challenge is to measure these muscles in a valid manner in clinical and research settings [4]. Measures of muscle strength cannot distinguish contributions from intrinsic and extrinsic muscles. The anatomical configuration of the intrinsic foot muscles, such as their small size and depth within the foot, limits electromyography studies to invasive intramuscular evaluations. Imaging modalities, such as ultrasound imaging (US) and magnetic resonance imaging (MRI), provide a non-invasive option to measure muscle morphology (size and shape) and composition, which are primary determinants of muscle function (force output) [5]. Threedimensional MRI is considered a gold standard for quantification of muscle morphology as it allows evaluation of the whole muscle (i.e. volume). This contrasts ultrasound, which typically involves 2-dimensional imaging and measures of cross-sectional area or thickness from transverse and longitudinal views. MRI also permits quantification of muscle composition (e.g. fat infiltration), which can also affect the force producing capacity of a muscle [6].

MRI of muscle of the foot has been used to evaluate intrinsic foot muscle morphology in several patient populations, such as individuals with diabetes [7-17], plantar heel pain $[18,19]$, foot pain $[20,21]$, CharcotMarie-Tooth [22, 23], and chronic ankle instability [24, 25 ], as well as to evaluate the effect of interventions such as physical therapy [24], footwear [26, 27] and foot exercise [28-30]. Table 1 provides a summary of published methods from studies that have evaluated intrinsic foot muscle morphology and composition. Previous studies have used a variety of methods, that vary in terms of field strengths from $0.5 \mathrm{~T}(0.5 \mathrm{~T})$ to $3 \mathrm{~T}$ and acquisition techniques (e.g. T1-, T2- and proton-density-weighted; gradient echo, spin echo and other sequences).

Of studies of intrinsic foot muscle morphology (i.e. volume, cross-sectional area and/or thickness), some have measured the intrinsic foot muscles as a group (i.e. total contractile tissue volume) [7, 8, 12-15, 17-19, 26], whereas others report individual muscles (e.g. abductor hallucis, flexor hallucis brevis, etc.) $[20,24,25,27-30$, 31] or muscle regions (e.g. muscle tissue under the first metatarsal head, medial/central/lateral muscle groups) $[9,16,32,33]$. Few studies have evaluated intrinsic foot muscle composition [10,11, 13, 14, 16, 20-23] and most have used qualitative grading scales of fatty atrophy, such as the five-point Goutellier scale [10, 11, 16, 20$22]$. Only two studies $[13,14]$ have quantified intermuscular adipose tissue volume, however this was performed for the intrinsic foot muscles as a whole, and not individual muscles. Low image resolution has limited the accuracy of segmentation of the architecturally complex intrinsic foot muscle group [12, 29, 32, 33].

Advanced MRI technologies and techniques, such as ultra-high-field scanners and chemical-shift sequences, offer the opportunity to enhance current evaluation and understanding of intrinsic foot muscle morphology. With double the signal-to-noise-ratio of 3T, imaging at 7T offers greater precision and accuracy towards quantification of intrinsic foot muscle morphology, particularly the segmentation of individual muscles and has not yet been realised. Chemical-shift MRI (e.g. DIXON [Siemens], IDEAL [General Electric], mDIXON [Phillips], FatSep $^{\text {тм }}$ [Hitachi], or WFS [Toshiba]) produces wateronly and fat-only images from dual-echo and/or multiecho acquisitions, which permits accurate quantification of muscle composition (e.g. percentage muscle fat infiltration). The aim of this paper is to provide a proof-ofconcept method for the measurement of intrinsic foot muscle morphology and composition with high-field MRI. As part of a series of papers [34, 35], here we also 
Table 1 A non-systematic summary of methods across studies that describe intrinsic foot muscle (IFM) morphology using magnetic resonance imaging (MRI)

\begin{tabular}{|c|c|c|c|c|c|c|c|}
\hline Citation & Reliability & MRI sequence & Slice selection & $\begin{array}{l}\text { Muscle of } \\
\text { interest }\end{array}$ & ROI selection & $\begin{array}{l}\text { Fat } \\
\text { detection }\end{array}$ & Measure \\
\hline $\begin{array}{l}\text { Andersen } \\
\text { et al., } 2004 \\
\text { [7] }\end{array}$ & Not reported & $\begin{array}{l}\text { 1T; T1-weighted } \\
\text { spin echo; } 4 \text { mm } \\
\text { slice thickness; } \\
\text { slice interval } \\
10 \text { mm }\end{array}$ & $\begin{array}{l}\text { All slices, the first } \\
\text { section being } \\
\text { randomly placed } \\
\text { within the first } \\
\text { interslice 10-mm } \\
\text { interval }\end{array}$ & $\begin{array}{l}\text { All, as a single } \\
\text { group }\end{array}$ & $\begin{array}{l}\text { Semi-automated } \\
\text { user set pixel } \\
\text { intensity threshold, } \\
\text { stereological point- } \\
\text { counting method }\end{array}$ & N/A & $\begin{array}{l}\text { Muscle volume } \\
\text { (expressed as a } \\
\text { percentage of the } \\
\text { value of the } \\
\text { matched control } \\
\text { participant) }\end{array}$ \\
\hline $\begin{array}{l}\text { Andreassen } \\
\text { et al., } 2009 \\
\text { [8] }\end{array}$ & Not reported & $\begin{array}{l}\text { 1.5T; T1-weighted } \\
\text { fast spin echo; } \\
4 \mathrm{~mm} \text { slice } \\
\text { thickness }\end{array}$ & All slices & $\begin{array}{l}\text { All, as a single } \\
\text { group }\end{array}$ & $\begin{array}{l}\text { Semi-automated } \\
\text { user set pixel } \\
\text { intensity threshold, } \\
\text { stereological point- } \\
\text { counting method }\end{array}$ & $\mathrm{N} / \mathrm{A}$ & $\begin{array}{l}\text { Muscle volume } \\
\left(\mathrm{cm}^{3}\right)\end{array}$ \\
\hline $\begin{array}{l}\text { Brash et al., } \\
1999 \text { [9] }\end{array}$ & Not reported & $\begin{array}{l}0.5 \mathrm{~T} ; \mathrm{T} 1 \text {-weighted } \\
\text { gradient-echo } \\
\text { and T2-weighted } \\
\text { spin echo }\end{array}$ & $\begin{array}{l}\text { Sagittal section } \\
\text { through first } \\
\text { metatarsal head }\end{array}$ & $\begin{array}{l}\text { Muscle tissue } \\
\text { under the first } \\
\text { metatarsal } \\
\text { head }\end{array}$ & Not described & $\mathrm{N} / \mathrm{A}$ & $\begin{array}{l}\text { Percentage of CSA } \\
\text { that was } \\
\text { magnetization } \\
\text { transfer active }\end{array}$ \\
\hline $\begin{array}{l}\text { Bus et al., } \\
2002[12]\end{array}$ & Not reported & $\begin{array}{l}\text { 3T; T2-weighted } \\
\text { fast spin echo; } \\
3 \mathrm{~mm} \text { slice thick- } \\
\text { ness; } 0.15 \mathrm{~mm} \\
\text { interslice gap; ac- } \\
\text { quisition time } \\
30 \text { min }\end{array}$ & $\begin{array}{l}2 \text { datasets collected } \\
\text { in frontal plane; } 1 \text { st } \\
\text { dataset } 40-46 \text { slices } \\
\text { from mid-tarsal joint } \\
\text { proximally \& distal } \\
\text { IP of 2nd toe dis- } \\
\text { tally; } 2 \text { nd dataset } 6 \\
\text { slices from distal } \\
\text { metatarsal region; } \\
\text { one slice through } \\
\text { head } 5 \text { th metatarsal } \\
\text { selected for quanti- } \\
\text { tative analysis }\end{array}$ & $\begin{array}{l}\text { All, as a single } \\
\text { group }\end{array}$ & $\begin{array}{l}\text { Semi-automated } \\
\text { signal intensity } \\
\text { levels using CCHIPS } \\
\text { software, verified by } \\
\text { visual inspection }\end{array}$ & $\mathrm{N} / \mathrm{A}$ & $\begin{array}{l}\text { Muscle CSA } \\
\text { (expressed as a } \\
\text { percentage of total } \\
\text { foot CSA) }\end{array}$ \\
\hline $\begin{array}{l}\text { Bus et al., } \\
2006[10]\end{array}$ & $\begin{array}{l}\text { Intra-rater } \\
\text { reliability; } 4 \\
\text { weeks between } \\
\text { measures; } \\
\text { weighted } \\
\text { kappa }=0.94\end{array}$ & $\begin{array}{l}\text { 1.5T; T1-weighted } \\
\text { spin echo; slice } \\
\text { thickness } 3 \mathrm{~mm} \text {; } \\
0.9 \mathrm{~mm} \text { interslice } \\
\text { gap }\end{array}$ & $\begin{array}{l}\text { Sagittal plane } \\
\text { images oriented } \\
\text { parallel to long axis } \\
\text { 2nd metatarsal and } \\
\text { perpendicular to } \\
\text { sole of the foot - } 19 \\
\text { slices acquired } \\
\text { between } 1 \text { st and } \\
\text { 5th metatarsal } \\
\text { heads; coronal plane } \\
\text { images oriented } \\
\text { perpendicular to } \\
\text { sagittal plane } \\
\text { images - } 20 \text { slices } \\
\text { between proximal } \\
\text { phalanx and } \\
\text { cuneiforms; one } \\
\text { slice through head } \\
\text { 5th metatarsal } \\
\text { selected for analysis }\end{array}$ & $\begin{array}{l}\text { All, as a single } \\
\text { group }\end{array}$ & Visual inspection & $\begin{array}{l}\text { Semi- } \\
\text { quantitative } \\
\text { 5-point scale }\end{array}$ & $\begin{array}{l}\text { Fatty atrophy: } 0= \\
\text { healthy muscle or } \\
\text { no atrophy; } 1=\text { mild } \\
\text { atrophy; } 2= \\
\text { moderate atrophy; } \\
3=\text { severe atrophy; } \\
4=\text { almost no or no } \\
\text { muscle tissue visible }\end{array}$ \\
\hline $\begin{array}{l}\text { Bus et al., } \\
2009[11]\end{array}$ & $\begin{array}{l}\text { Cited Bus et al., } \\
2006 \\
\text { (Intra-rater } \\
\text { reliability; } \\
\text { weighted } \\
\text { kappa }=0.94 \text { ) }\end{array}$ & $\begin{array}{l}\text { 1.5T; T1-weighted } \\
\text { spin echo; slice } \\
\text { thickness } 3 \mathrm{~mm} ; \\
0.9 \mathrm{~mm} \text { interslice } \\
\text { gap }\end{array}$ & $\begin{array}{l}\text { Sagittal plane } \\
\text { images - } 19 \text { slices } \\
\text { acquired between } \\
\text { 1st and 5th } \\
\text { metatarsal heads; } \\
\text { coronal plane } \\
\text { images - } 20 \text { slices } \\
\text { between proximal } \\
\text { phalanx and } \\
\text { cuneiforms; one } \\
\text { slice through head } \\
\text { 5th metatarsal } \\
\text { selected for analysis }\end{array}$ & $\begin{array}{l}\text { All, as a single } \\
\text { group }\end{array}$ & Visual inspection & $\begin{array}{l}\text { Semi- } \\
\text { quantitative } \\
\text { 5-point scale }\end{array}$ & $\begin{array}{l}\text { Fatty atrophy: } 0= \\
\text { healthy muscle } \\
\text { tissue or no } \\
\text { atrophy; } 1=\text { mild } \\
\text { atrophy; } \\
2=\text { moderate } \\
\text { atrophy; } 3 \\
=\text { severe atrophy; } 4 \\
= \\
\text { almost complete or } \\
\text { complete loss } \\
\text { of muscle tissue }\end{array}$ \\
\hline $\begin{array}{l}\text { Chang } \\
\text { et al., } 2012\end{array}$ & $\begin{array}{l}\text { Intra-rater } \\
\text { reliability; one }\end{array}$ & $\begin{array}{l}\text { 1.5T; T1-weighted } \\
\text { spin echo; slice }\end{array}$ & $\begin{array}{l}\text { Frontal plane } \\
\text { images acquired }\end{array}$ & $\begin{array}{l}\text { All, as a single } \\
\text { group (but }\end{array}$ & $\begin{array}{l}\text { Interactive custom } \\
\text { software }\end{array}$ & $\mathrm{N} / \mathrm{A}$ & $\begin{array}{l}\text { Muscle volume } \\
\left(\mathrm{cm}^{3}\right)\end{array}$ \\
\hline
\end{tabular}


Table 1 A non-systematic summary of methods across studies that describe intrinsic foot muscle (IFM) morphology using magnetic resonance imaging (MRI) (Continued)

\begin{tabular}{|c|c|c|c|c|c|c|c|}
\hline Citation & Reliability & MRI sequence & Slice selection & $\begin{array}{l}\text { Muscle of } \\
\text { interest }\end{array}$ & ROI selection & $\begin{array}{l}\text { Fat } \\
\text { detection }\end{array}$ & Measure \\
\hline [18] & $\begin{array}{l}\text { image } \\
\text { processed } 5 \\
\text { times; } \\
\text { coefficient of } \\
\text { variation of } \\
\text { muscle CSA = } \\
1.3 \%\end{array}$ & $\begin{array}{l}\text { thickness } 4 \mathrm{~mm} ; \\
\text { contiguous slices; } \\
\text { acquisition time } \\
25 \text { min }\end{array}$ & $\begin{array}{l}\text { perpendicular to } \\
\text { plantar aspect of } \\
\text { foot; every image } \\
\text { from calcaneus } \\
\text { through to image } \\
\text { containing } \\
\text { maximum diameter } \\
\text { of sesamoid bones }\end{array}$ & excluding EDB) & $\begin{array}{l}\text { programmed in } \\
\text { Matlab; Semi- } \\
\text { automated user set } \\
\text { pixel intensity } \\
\text { threshold }\end{array}$ & & \\
\hline $\begin{array}{l}\text { Chen et al., } \\
2016 \text { [26] }\end{array}$ & $\begin{array}{l}\text { Cited Cheung } \\
\text { et al., } 2016 \text { (who } \\
\text { cited Chang } \\
\text { 2012) }\end{array}$ & $\begin{array}{l}\text { 1.5T; T1-weighted } \\
\text { spin echo; slice } \\
\text { thickness } 4 \mathrm{~mm} \text {; } \\
\text { contiguous slices }\end{array}$ & $\begin{array}{l}\text { Sagittal and frontal } \\
\text { plane acquisition; } \\
\text { entire length of foot }\end{array}$ & $\begin{array}{l}\text { All, as a single } \\
\text { group }\end{array}$ & $\begin{array}{l}\text { Segmented by } \\
\text { excluding all non- } \\
\text { contractile tissues in } \\
\text { Mimics software }\end{array}$ & $\mathrm{N} / \mathrm{A}$ & $\begin{array}{l}\text { Muscle volume } \\
\left(\mathrm{mm}^{3} / \mathrm{kg}\right)\end{array}$ \\
\hline $\begin{array}{l}\text { Cheung } \\
\text { et al., } 2016 \\
\text { [19] }\end{array}$ & $\begin{array}{l}\text { Cited Chang } \\
\text { et al., } 2012\end{array}$ & $\begin{array}{l}\text { 1.5T; T1-weighted; } \\
\text { Slice thickness } \\
4 \mathrm{~mm} \text {; contigu- } \\
\text { ous slices }\end{array}$ & $\begin{array}{l}\text { Images acquired } \\
\text { perpendicularly to } \\
\text { the plantar aspect } \\
\text { of the foot; entire } \\
\text { length of foot }\end{array}$ & $\begin{array}{l}\text { All, as a single } \\
\text { group }\end{array}$ & $\begin{array}{l}\text { Segmented by } \\
\text { excluding all non- } \\
\text { contractile tissues in } \\
\text { Mimics software }\end{array}$ & $\mathrm{N} / \mathrm{A}$ & $\begin{array}{l}\text { Muscle volume } \\
\left(\mathrm{mm}^{3} / \mathrm{kg}\right)\end{array}$ \\
\hline $\begin{array}{l}\text { Cheuy et al., } \\
2013 a \text { [13] }\end{array}$ & $\begin{array}{l}\text { Inter- and intra- } \\
\text { rater reliability; } 2 \\
\text { raters processed } \\
46 \text { slices with at } \\
\text { least } 14 \text { days } \\
\text { between mea- } \\
\text { sures; all ICCs > } \\
0.9\end{array}$ & $\begin{array}{l}\text { 3T; optimised to } \\
\text { fat; spin echo } \\
\text { pulse; slice } \\
\text { thickness } 3.5 \mathrm{~mm} \text {; } \\
\text { acquisition time } \\
\text { 9-12 min; }\end{array}$ & $\begin{array}{l}\text { Coronal plane } \\
\text { images acquired; } \\
\text { 35-65 slices; the } \\
\text { forefoot (mid- } \\
\text { metatarsal), the } \\
\text { midfoot } \\
\text { (tarsometatarsal } \\
\text { joint of the second } \\
\text { metatarsal), the } \\
\text { hindfoot } \\
\text { (talonavicular joint) }\end{array}$ & $\begin{array}{l}\text { Plantar side } \\
\text { muscles, as a } \\
\text { single group }\end{array}$ & $\begin{array}{l}\text { Signal intensity } \\
\text { threshold } \\
\text { automatically } \\
\text { identified with } \\
\text { optional manual } \\
\text { editing of borders } \\
\text { and thresholds as } \\
\text { required, Matlab } \\
\text { software }\end{array}$ & Quantitative & $\begin{array}{l}\text { Volume }\left(\mathrm{cm}^{3}\right) \text { : } \\
\text { Subcutaneous fat; } \\
\text { Lean muscle; } \\
\text { Intermuscular } \\
\text { adipose tissue }\end{array}$ \\
\hline $\begin{array}{l}\text { Cheuy et al., } \\
\text { 2013b [14] }\end{array}$ & Not reported & $\begin{array}{l}\text { 3T; spin echo } \\
\text { pulse; slice } \\
\text { thickness } 3.5 \mathrm{~mm} \text {; } \\
\text { acquisition time } \\
12 \mathrm{~min}\end{array}$ & $\begin{array}{l}\text { Coronal plane } \\
\text { images acquired; } 35 \\
\text { slices; talonavicular } \\
\text { joint to } \\
\text { tarsometatarsal joint }\end{array}$ & $\begin{array}{l}\text { Intrinsic foot } \\
\text { muscles } \\
\text { between the } \\
\text { talonavicular } \\
\text { and } \\
\text { tarsometatarsal } \\
\text { joints }\end{array}$ & $\begin{array}{l}\text { Signal intensity } \\
\text { threshold } \\
\text { automatically } \\
\text { identified with } \\
\text { optional manual } \\
\text { editing of borders } \\
\text { and thresholds as } \\
\text { required, Matlab } \\
\text { software }\end{array}$ & Quantitative & $\begin{array}{l}\text { Volume }\left(\mathrm{cm}^{3}\right) \text { : } \\
\text { Subcutaneous fat; } \\
\text { Lean muscle; } \\
\text { Intermuscular } \\
\text { adipose tissue; } \\
\text { Intrinsic foot muscle } \\
\text { ratio (ratio of } \\
\text { intermuscular } \\
\text { volume to lean } \\
\text { muscle volume }\end{array}$ \\
\hline $\begin{array}{l}\text { Feger et al., } \\
2016 \text { [25] }\end{array}$ & Not reported & $\begin{array}{l}\text { 3T; spiral gradient } \\
\text { echo; slice } \\
\text { thickness } 5 \mathrm{~mm} \text {; } \\
\text { acquisition time } \\
15 \text { min }\end{array}$ & $\begin{array}{l}\text { Axial slices; entire } \\
\text { foot }\end{array}$ & $\begin{array}{l}\text { ABH; ADDH-O; } \\
\text { ADDH-T; FHB; } \\
\text { ABDM; FDM; } \\
\text { EDB; FDB; QP, } \\
\text { Interosseous }\end{array}$ & $\begin{array}{l}\text { Manual } \\
\text { segmentation of } \\
\text { each muscle } \\
\text { perimeter on each } \\
\text { slice using custom } \\
\text { software written in } \\
\text { MatLab }\end{array}$ & $\mathrm{N} / \mathrm{A}$ & $\begin{array}{l}\text { Muscle volume } \\
\left(\mathrm{cm}^{3} / \mathrm{m} . \mathrm{kg}\right)\end{array}$ \\
\hline $\begin{array}{l}\text { Feger et al., } \\
2019 \text { [24] }\end{array}$ & $\begin{array}{l}\text { Cited Handsfield } \\
\text { et al., } 2014 \\
\text { (study of leg } \\
\text { muscle } \\
\text { segmentation; } \\
\text { inter-user vari- } \\
\text { ability reported } \\
\text { as acceptable at } \\
<0.6 \% \text { ) }\end{array}$ & $\begin{array}{l}\text { 3T; spiral gradient } \\
\text { echo; slice } \\
\text { thickness } 5 \mathrm{~mm} \text {; } \\
\text { acquisition time } \\
15 \text { min }\end{array}$ & $\begin{array}{l}\text { Axial slices were } \\
\text { obtained in sets of } \\
20 \text { contiguous } \\
\text { images from just } \\
\text { posterior to the } \\
\text { calcaneus anteriorly } \\
\text { through the entire } \\
\text { foot. }\end{array}$ & $\begin{array}{l}\text { ABH; ADDH-O; } \\
\text { ADDH-T; FHB; } \\
\text { ABDM; FDM; } \\
\text { EDB; FDB; QP, } \\
\text { Interosseous }\end{array}$ & $\begin{array}{l}\text { Manual } \\
\text { segmentation of } \\
\text { each muscle } \\
\text { perimeter on each } \\
\text { slice using custom } \\
\text { software written in } \\
\text { MatLab }\end{array}$ & $\mathrm{N} / \mathrm{A}$ & $\begin{array}{l}\text { Muscle volume } \\
\left(\mathrm{cm}^{3} / \mathrm{m} . \mathrm{kg}\right)\end{array}$ \\
\hline $\begin{array}{l}\text { Gallardo } \\
\text { et al., } 2006 \\
\text { [23] }\end{array}$ & Not reported & $\begin{array}{l}\text { 1.5T; T1-weighted } \\
\text { fast spin-echo } \\
\text { and fat-supressed } \\
\text { proton density-T2 } \\
\text { weighted fast } \\
\text { spin-echo in both } \\
\text { planes; }\end{array}$ & $\begin{array}{l}\text { Coronal and axial } \\
\text { planes }\end{array}$ & All & Visual inspection & Qualitative & $\begin{array}{l}\text { Presence of signal } \\
\text { intensity alterations } \\
\text { including muscle } \\
\text { oedema, fatty } \\
\text { infiltration and } \\
\text { abnormal } \\
\text { enhancement }\end{array}$ \\
\hline
\end{tabular}


Table 1 A non-systematic summary of methods across studies that describe intrinsic foot muscle (IFM) morphology using magnetic resonance imaging (MRI) (Continued)

\begin{tabular}{|c|c|c|c|c|c|c|c|}
\hline Citation & Reliability & MRI sequence & Slice selection & $\begin{array}{l}\text { Muscle of } \\
\text { interest }\end{array}$ & ROI selection & $\begin{array}{l}\text { Fat } \\
\text { detection }\end{array}$ & Measure \\
\hline & & $\begin{array}{l}\text { slice thickness } \\
10 \mathrm{~mm} \text { with 0.5- } \\
1.0 \mathrm{~mm} \text { slice gap; } \\
\text { coronal plane } \\
\text { slice thickness 4- } \\
5 \mathrm{~mm} \text { with 0.5- } \\
1.0 \mathrm{~mm} \text { slice gap }\end{array}$ & & & & & \\
\hline $\begin{array}{l}\text { Gooding } \\
\text { et al., } 2016 \\
{[29]}\end{array}$ & Not reported & $\begin{array}{l}\text { 3T; turbo spin } \\
\text { echo; slice } \\
\text { thickness } 10 \mathrm{~mm} \text {; } \\
0 \mathrm{~mm} \text { interslice } \\
\text { gap; acquisition } \\
\text { time } 7 \text { min }\end{array}$ & $\begin{array}{l}\text { From most posterior } \\
\text { aspect calcaneus to } \\
\text { the toes; for each } \\
\text { muscle the series of } \\
3 \text { contiguous slices } \\
\text { that provided the } \\
\text { largest CSA used for } \\
\text { analysis }\end{array}$ & $\begin{array}{l}\text { ABH, FDB, } \\
\text { ABDM, QP, } \\
\text { FDM, ADDH-O, } \\
\text { FHB, Interosse- } \\
\text { ous \& lumbri- } \\
\text { cals (together) }\end{array}$ & $\begin{array}{l}\text { Each muscle } \\
\text { manually outlined; } \\
\text { pixel-by-pixel count } \\
\text { based on active } \\
\text { range (any pixel } \\
\text { that exceeded the } \\
\text { lower threshold) }\end{array}$ & N/A & $\begin{array}{l}\text { Percentage increase } \\
\text { in muscle activation } \\
\text { (pre to post } \\
\text { exercise) }\end{array}$ \\
\hline $\begin{array}{l}\text { Green \& } \\
\text { Briggs, } 2013 \\
{[32]}\end{array}$ & Not reported & $\begin{array}{l}\text { Magnet strength } \\
\text { not specified; T1- } \\
\text { \& proton density } \\
\text { weighted; slice } \\
\text { thickness not } \\
\text { specified; } \\
\text { interslice gap } \\
5 \mathrm{~mm} \text {; }\end{array}$ & $\begin{array}{l}\text { Coronal plane } \\
\text { images acquired } \\
\text { with the plane tilted } \\
\text { antero-superiorly to } \\
\text { lie perpendicular to } \\
\text { the long axis of the } \\
\text { second metatarsal }\end{array}$ & $\begin{array}{l}5 \text { groups: } \\
\text { (1) Medial - } \\
\text { ABH, FHB; (2) } \\
\text { ADDH; (3) } \\
\text { Central - FDB, } \\
\text { QP, lumbricals; } \\
\text { (4) } \\
\text { Interosseous; } \\
\text { (5) Lateral - } \\
\text { ABDM, FDM }\end{array}$ & $\begin{array}{l}\text { Measured using a } \\
\text { freehand cursor to } \\
\text { trace around the } \\
\text { muscle groups }\end{array}$ & N/A & $\begin{array}{l}\text { Maximum muscle } \\
\mathrm{CSA}\left(\mathrm{cm}^{2}\right)\end{array}$ \\
\hline $\begin{array}{l}\text { Greenman } \\
\text { et al., } 2005 \\
{[15]}\end{array}$ & Not reported & $\begin{array}{l}\text { 3T; T2-weighted } \\
\mathrm{H} \text { spin echo: } \\
\text { thickness } 2.5 \mathrm{~mm} \text {; } \\
\text { acquisition time } \\
6 \text { min } 24 \mathrm{~s} \text {; } \\
\text { RARE pulse: slice } \\
\text { thickness } 25 \mathrm{~mm} ; \\
\text { acquisition time } \\
4 \text { min }\end{array}$ & $\begin{array}{l}\text { Axial plane slices; } 10 \\
\text { contiguous } \\
\text { locations }\end{array}$ & $\begin{array}{l}\text { All, as a single } \\
\text { group }\end{array}$ & $\begin{array}{l}\text { Interactive data } \\
\text { language software; } \\
\text { an outline of the } \\
\text { muscle tissue and } \\
\text { reference standard } \\
\text { was created using a } \\
\text { contour mapping } \\
\text { function set to a } \\
\text { single level that was } \\
\text { equal to the noise } \\
\text { threshold value; } \\
\text { count of pixels that } \\
\text { represented signal } \\
\text { from foot tissues }\end{array}$ & N/A & $\begin{array}{l}\text { Muscle area- to } \\
\text {-total area ratio }\end{array}$ \\
\hline $\begin{array}{l}\text { Kurihara } \\
\text { et al., } 2014 \\
{[33]}\end{array}$ & Not reported & $\begin{array}{l}\text { 1.5T; T1-weighted } \\
\text { fast spin echo; } \\
\text { slice thickness } \\
4 \mathrm{~mm} \text {; acquisition } \\
\text { time } ~ 9 \mathrm{~min}\end{array}$ & $\begin{array}{l}\text { Whole foot } \\
\text { (sesamoids to } \\
\text { calcaneal } \\
\text { tuberosity); } \\
\text { contiguous slices; } \\
\text { acquired } \\
\text { perpendicular to } \\
\text { plantar aspect of } \\
\text { foot; image at the } \\
\text { MTP joint that was } \\
\text { near 20\% } \\
\text { longitudinal foot } \\
\text { length was selected } \\
\text { for analysis }\end{array}$ & $\begin{array}{l}3 \text { muscle } \\
\text { groups: 1) } \\
\text { Medial - FHB, } \\
\text { FDB, QP, ABH } \\
\text { and lumbricals; } \\
\text { 2) ADDH; 3) } \\
\text { Lateral - } \\
\text { ABDM, FDB, } \\
\text { interosseus }\end{array}$ & $\begin{array}{l}\text { Manual; SliceOmatic } \\
\text { software; excluded } \\
\text { non-contractile tis- } \\
\text { sues where possible }\end{array}$ & N/A & Muscle CSA $\left(\mathrm{cm}^{2)}\right.$ \\
\hline $\begin{array}{l}\text { Lin et al., } \\
2016 \text { [16] }\end{array}$ & Not reported & $\begin{array}{l}\text { 3T; T1-weighted } \\
{ }^{1} \mathrm{H} \text { spin: slice } \\
\text { thickness } 2.5 \mathrm{~mm} \text {; } \\
\text { acquisition time } \\
6 \text { min } 24 \mathrm{~s} ;{ }^{31} \mathrm{P} \text { - } \\
\text { RARE: slice thick- } \\
\text { ness } 25 \mathrm{~mm} \text {; scan } \\
\text { time } 4 \mathrm{~min}\end{array}$ & $\begin{array}{l}\text { Acquired in a plane } \\
\text { perpendicular to } \\
\text { longitudinal } \\
\text { direction of foot } \\
\text { through the } \\
\text { metatarsal head } \\
\text { region; } 10 \\
\text { contiguous; } \\
\text { Selected for analysis: } \\
\text { level of the 5th }\end{array}$ & $\begin{array}{l}3 \text { muscle } \\
\text { regions: } \\
\text { (1) FHB medial } \\
\text { head; (2) } \\
\text { ADDH, FHB } \\
\text { lateral head, } \\
\text { lumbricals; (3) } \\
\text { Interosseous, } \\
\text { FDM, ABDM }\end{array}$ & $\begin{array}{l}\text { Manually outlined } \\
\text { using tracing tool in } \\
\text { OsiriX software; } \\
\text { pixel threshold } \\
\text { technique }\end{array}$ & $\begin{array}{l}\text { Semi- } \\
\text { quantitative } \\
\text { 5-point scale }\end{array}$ & $\begin{array}{l}\text { Ratio }\left({ }^{31} \mathrm{P} /{ }^{1} \mathrm{H}\right) \text { of the } \\
\text { area of viable } \\
\text { muscle tissue to } \\
\text { total area outlined; } \\
\text { Fatty atrophy: } 0= \\
\text { healthy muscle with } \\
\text { no atrophy; } 1=\text { mild } \\
\text { atrophy; } 2= \\
\text { moderate atrophy; } 3 \\
=\end{array}$ \\
\hline
\end{tabular}


Table 1 A non-systematic summary of methods across studies that describe intrinsic foot muscle (IFM) morphology using magnetic resonance imaging (MRI) (Continued)

\begin{tabular}{|c|c|c|c|c|c|c|c|}
\hline Citation & Reliability & MRI sequence & Slice selection & $\begin{array}{l}\text { Muscle of } \\
\text { interest }\end{array}$ & ROI selection & $\begin{array}{l}\text { Fat } \\
\text { detection }\end{array}$ & Measure \\
\hline & & & $\begin{array}{l}\text { metatarsal head } \\
\text { from T1-weighted } \\
\text { images }\end{array}$ & & & & $\begin{array}{l}\text { severe atrophy; } 4= \\
\text { almost no or no } \\
\text { muscle tissue } \\
\text { visible. }\end{array}$ \\
\hline $\begin{array}{l}\text { Miller et al., } \\
2014 \text { [27] }\end{array}$ & $\begin{array}{l}\text { Intra-rater } \\
\text { reliability; five } \\
\text { measurements } \\
\text { on each muscle } \\
\text { over multiple } \\
\text { days; mean } \\
\text { measurement } \\
\text { relative error } \\
0.2-4.3 \%\end{array}$ & $\begin{array}{l}\text { 1.5T; T2 turbo } \\
\text { spin echo fat } \\
\text { saturation; slice } \\
\text { thickness } 5 \mathrm{~mm}\end{array}$ & $\begin{array}{l}\text { Coronal, sagittal, } \\
\text { axial scans; entire } \\
\text { foot; muscle } \\
\text { measurement from } \\
\text { axial scan }\end{array}$ & $\begin{array}{l}\mathrm{ABH} ; \mathrm{FDB} \\
\mathrm{ABDM}\end{array}$ & $\begin{array}{l}\text { Manually traced in } \\
\text { ImageJ }\end{array}$ & $N / A$ & $\begin{array}{l}\text { Muscle CSA }\left(\mathrm{mm}^{2}\right) \\
\text { Muscle volume } \\
\left(\mathrm{mm}^{3}\right) \\
\text { log normalised to } \\
\text { foot length }\end{array}$ \\
\hline $\begin{array}{l}\text { Pelayo- } \\
\text { Negro et al., } \\
2014 \text { [22] }\end{array}$ & Not reported & $\begin{array}{l}\text { 1.5T; T1-weighted } \\
\text { fast spin-echo; fat } \\
\text { suppressed pro- } \\
\text { ton density-T2-- } \\
\text { weighted fast } \\
\text { spin-echo }\end{array}$ & $\begin{array}{l}\text { Slice thickness not } \\
\text { specified; axial and } \\
\text { coronal planes }\end{array}$ & $\begin{array}{l}\text { All, as a single } \\
\text { group }\end{array}$ & Visual inspection & $\begin{array}{l}\text { Semi- } \\
\text { quantitative; } \\
\text { 5-point scale }\end{array}$ & $\begin{array}{l}\text { Fatty infiltration: } 0= \\
\text { no evidence of fatty } \\
\text { infiltration; } 1=\text { some } \\
\text { fatty streaks; } 2 \text { = fat } \\
\text { evident but } \\
\text { less extensive than } \\
\text { muscle; } 3=\text { fat } \\
\text { equal to muscle; } \\
\text { and stage } 4=\text { fat } \\
\text { more extensive } \\
\text { than muscle. }\end{array}$ \\
\hline $\begin{array}{l}\text { Recht et al., } \\
2007 \text { [21] }\end{array}$ & Not reported & $\begin{array}{l}0.2 \text { to } 1.5 \mathrm{~T} \text {; T1- } \\
\text { and T2-weighted } \\
\text { (with or without } \\
\text { fat suppression) } \\
\text { in coronal; STIR or } \\
\text { T2-weighted in } \\
\text { sagittal }\end{array}$ & All images & ABDM & Visual inspection & $\begin{array}{l}\text { Semi- } \\
\text { quantitative; } \\
\text { 4-point scale }\end{array}$ & $\begin{array}{l}\text { Fatty atrophy: grade } \\
0= \\
\text { no fat or minimal } \\
\text { fatty streaks; } 1= \\
\text { increased } \\
\text { fat within the } \\
\text { muscle but greater } \\
\text { amount of muscle; } \\
2=\text { equal amounts } \\
\text { of fat and muscle; } \\
3=\text { greater amount } \\
\text { of fat than } \\
\text { muscle }\end{array}$ \\
\hline $\begin{array}{l}\text { Savnik et al., } \\
2000 \text { [31] }\end{array}$ & Not reported & $\begin{array}{l}\text { 1.5T; T1-weighted } \\
\text { spin echo; } \\
\text { T2-weighted spin } \\
\text { echo and STIR; } \\
\text { slice thickness 3- } \\
4 \text { mm; interslice } \\
\text { gap 0.3-0.4 mm }\end{array}$ & $\begin{array}{l}\text { Sagittal images } \\
\text { acquired; coronal } \\
\text { reformatted images } \\
\text { used for } \\
\text { measurement }\end{array}$ & QP; FDB; EH & $\begin{array}{l}\text { Measured using } \\
\text { region-of-interest } \\
\text { area function }\end{array}$ & $N / A$ & $\begin{array}{l}\text { Muscle largest } \\
\text { diameter and } \\
\text { transverse area }\end{array}$ \\
\hline $\begin{array}{l}\text { Schmid } \\
\text { et al., } 2009 \\
\text { [20] }\end{array}$ & $\begin{array}{l}\text { Intra-rater } \\
\text { reliability CSA } \\
\text { measures; 20\% } \\
\text { of measures } \\
\text { repeated; } \\
\text { ICCs > } 0.9 \\
\text { Inter-rater } \\
\text { reliability fatty } \\
\text { atrophy score; } \\
\text { kappa } 0.33 \text { to } \\
0.68\end{array}$ & $\begin{array}{l}\text { 1.5T; T1- and T2- } \\
\text { weighted; Slice } \\
\text { thickness 3- } \\
3.5 \mathrm{~mm}\end{array}$ & $\begin{array}{l}\text { Coronal images; CSA } \\
\text { measures at the } \\
\text { level where the } \\
\text { bony insertion of } \\
\text { the tibiocalcaneal } \\
\text { ligament at the } \\
\text { calcaneus was best } \\
\text { visualised }\end{array}$ & $\begin{array}{l}\text { ABDM; FDB; } \\
\mathrm{ABH} ; \mathrm{QP}\end{array}$ & $\begin{array}{l}\text { Visual inspection } \\
\text { and measurements } \\
\text { using OsiriX } \\
\text { software }\end{array}$ & $\begin{array}{l}\text { Semi- } \\
\text { quantitative } \\
\text { 3-point scale }\end{array}$ & $\begin{array}{l}\text { Muscle CSA }\left(\mathrm{cm}^{2}\right) \\
\text { Fatty muscle } \\
\text { atrophy: } 0=\text { normal } \\
\text { muscle; } 1=\text { mild } \\
\text { fatty atrophy with } \\
\text { more muscle than } \\
\text { fat; } 2 \text { = substantial } \\
\text { fatty atrophy } \\
\text { with more fat than } \\
\text { muscle or equal } \\
\text { parts fat and muscle }\end{array}$ \\
\hline $\begin{array}{l}\text { Severinsen } \\
\text { et al., } 2007 \\
{[17]}\end{array}$ & Not reported & $\begin{array}{l}\text { 1.5T; } \mathrm{T} 1 \text { spin } \\
\text { echo; slice } \\
\text { thickness } 1.5 \mathrm{~mm} \text {; } \\
\text { inter-slice interval } \\
10 \mathrm{~mm}\end{array}$ & $\begin{array}{l}\text { The first section } \\
\text { being randomly } \\
\text { placed within the } \\
\text { first interslice } \\
\text { interval }\end{array}$ & $\begin{array}{l}\text { All, as a single } \\
\text { group }\end{array}$ & $\begin{array}{l}\text { Semi-automated } \\
\text { user set signal } \\
\text { intensity threshold, } \\
\text { stereological point- } \\
\text { counting technique }\end{array}$ & N/A & $\begin{array}{l}\text { Muscle volume } \\
\left(\mathrm{mm}^{3}\right)\end{array}$ \\
\hline $\begin{array}{l}\text { Taddei } \\
\text { et al., } 2018\end{array}$ & Not reported & $\begin{array}{l}\text { 1.5T; T1-weighted } \\
\text { spin-echo; slice }\end{array}$ & $\begin{array}{l}\text { Images acquired } \\
\text { perpendicular to the }\end{array}$ & $\begin{array}{l}\mathrm{ABH} ; \mathrm{ABDM} ; \\
\mathrm{FHB} ; \mathrm{FDB}\end{array}$ & $\begin{array}{l}\text { Measured by } \\
\text { ImageJ planimeter }\end{array}$ & N/A & Muscle CSA $\left(\mathrm{mm}^{2}\right)$ \\
\hline
\end{tabular}


Table 1 A non-systematic summary of methods across studies that describe intrinsic foot muscle (IFM) morphology using magnetic resonance imaging (MRI) (Continued)

\begin{tabular}{|c|c|c|c|c|c|c|c|}
\hline Citation & Reliability & MRI sequence & Slice selection & $\begin{array}{l}\text { Muscle of } \\
\text { interest }\end{array}$ & ROI selection & $\begin{array}{l}\text { Fat } \\
\text { detection }\end{array}$ & Measure \\
\hline [28] & & $\begin{array}{l}\text { thickness } 4 \mathrm{~mm} \text {; } \\
\text { contiguous }\end{array}$ & $\begin{array}{l}\text { plantar aspect of } \\
\text { the foot; between } \\
\text { the most proximal } \\
\text { and most distal } \\
\text { images in which } \\
\text { every intrinsic foot } \\
\text { muscle is visible }\end{array}$ & & $\begin{array}{l}\text { software for each } \\
\text { muscle at each slice }\end{array}$ & & \\
\hline $\begin{array}{l}\text { Taddei } \\
\text { et al., } 2020 \\
{[30]}\end{array}$ & Not reported & $\begin{array}{l}\text { 1.5T; T1-weighted } \\
\text { spin-echo; slice } \\
\text { thickness } 4 \mathrm{~mm} \text {; } \\
\text { contiguous }\end{array}$ & $\begin{array}{l}\text { Images acquired } \\
\text { perpendicular to the } \\
\text { plantar aspect of } \\
\text { the foot; between } \\
\text { the most proximal } \\
\text { and most distal } \\
\text { images in which } \\
\text { every intrinsic foot } \\
\text { muscle is visible }\end{array}$ & $\begin{array}{l}\mathrm{ABH} ; \mathrm{ABDM} ; \\
\mathrm{FHB} ; \mathrm{FDB}\end{array}$ & $\begin{array}{l}\text { Measured by } \\
\text { ImageJ planimeter } \\
\text { software for each } \\
\text { muscle at each slice }\end{array}$ & N/A & $\begin{array}{l}\text { Muscle CSA }\left(\mathrm{mm}^{2}\right) \\
\text { Muscle volume } \\
\left(\mathrm{cm}^{2}\right)\end{array}$ \\
\hline
\end{tabular}

$A B H$ Abductor hallucis, $A B D M$ Abductor digiti minimi, $A D D H$ Adductor hallucis (oblique and transverse heads combined), $A D D H-O$ Adductor hallucis oblique head, $A D D H-T$ Adductor hallucis transverse head, EDB Extensor digitorum brevis, EH Extensor hallucis, FDB Flexor digitorum brevis, FDM Flexor digiti minimi, FHB Flexor hallucis brevis, QP Quadratus plantae, CSA Cross-sectional area, N/A Not applicable

aim to promote improved reporting of image acquisition and image measurement procedures.

\section{Methods}

One healthy female (39 years, mass $65 \mathrm{~kg}$, height $1.73 \mathrm{~m}$, body mass index 21.7, foot length $26.2 \mathrm{~cm}$ ) was recruited by convenience to participate in the proof-of-concept study. Ethical approval was provided by The University of Queensland Human Research Ethics Committee (\#2, 018,001,150). The participant provided informed written consent and attended The University of Queensland's Centre for Advanced Imaging for a single imaging session. The participant underwent MRI of the whole foot on two different magnetic resonance scanners: a 7T MAGNETOM (Siemens AG, Erlangen, Germany) magnetic resonance scanner with a knee coil (QED knee coil $1 T X / 28 R x$ ) and a 3T MAGNETOM Prisma (Siemens AG, Erlangen, Germany) magnetic resonance scanner with a knee coil ( $\mathrm{Tx} / \mathrm{Rx}$ 15-channel knee coil). Patient positioning was standardised for both scanners (Fig. 1). To enable positioning of the foot within the coil, and allow maximal visualisation of the foot on the MR images, the participant was positioned in prone with the knee supported in slight flexion by a foam pad under the tibia, and the ankle and foot supported in plantar flexion within the receiver coil. Foam padding was placed between the dorsum of the foot and the receiver coil. The position of the foot and ankle was supported by foam wedges and sandbags to minimise movement.

A T1-weighted VIBE - radio-frequency spoiled 3D steady state GRE - sequence of the whole foot was acquired on each scanner. For comparability, acquisition time was standardised (7T: 4 minutes 50 seconds; 3T: 4 minutes 47 seconds). $7 \mathrm{~T}$ acquisition parameters were: repetition time $11 \mathrm{~ms}$; echo time $2.04 \mathrm{~ms}$; flip angle 3 degrees; field of view $175 \times 224 \mathrm{~mm}^{2}$; acquired voxel dimensions $0.50 \times 0.50 \mathrm{~mm}^{2} ; 0.50 \mathrm{~mm}$ contiguous slices; 176 slices; Bandwidth $429 \mathrm{~Hz} / \mathrm{Px})$. 3T acquisition parameters were: repetition time $15 \mathrm{~ms}$; echo time $2.45 \mathrm{~ms}$; flip angle 10 degrees; field of view $220 \times 220 \mathrm{~mm}^{2}$; acquired voxel dimensions $0.60 \times 0.60 \mathrm{~mm}^{2} ; 0.60 \mathrm{~mm}$ contiguous slices; 144 slices; Bandwidth $430 \mathrm{~Hz} / \mathrm{Px}$ ). To demonstrate the full capability of the 7T scanner, a highresolution fat/water separation image was also acquired using a 3D 2-point DIXON sequence (repetition time $11 \mathrm{~ms}$; echo time $3.06 \mathrm{~ms}$ and $5.61 \mathrm{~ms}$; flip angle $3 \mathrm{de}-$ grees; field of view $111 \times 223 \mathrm{~mm}^{2}$; acquired voxel dimensions $0.38 \times 0.38 \mathrm{~mm}^{2} ; 0.38 \mathrm{~mm}$ contiguous slices; 256 slices; bandwidth $434 \mathrm{~Hz} / \mathrm{Px}$; acquisition time $11 \mathrm{~min} 12 \mathrm{~s}$ ).

For comparison of 3T and 7T image resolution, images were imported to OsiriX MD (Pixmeo SARL, Switzerland) and coronal plane images from each T1weighted series were selected at five anatomical locations (Fig. 2): (A) the level of the sustentaculum tali, (B) immediately distal to the talo-navicular joint, $(\mathrm{C})$ immediately distal to the first tarso-metatarsal joints, (D) the mid-shaft of the first metatarsal and (E) the distal shaft of the first metatarsal (up to the metatarsal head). These locations were selected to provide example images from the rearfoot, midfoot and forefoot regions, as well as to include visualisation of all intrinsic foot muscles.

For the quantitative assessment of intrinsic foot muscle structure and composition, muscle volume and percentage muscle fat infiltration were measured from the 7T fat-water images using 3D Slicer software [36]. 


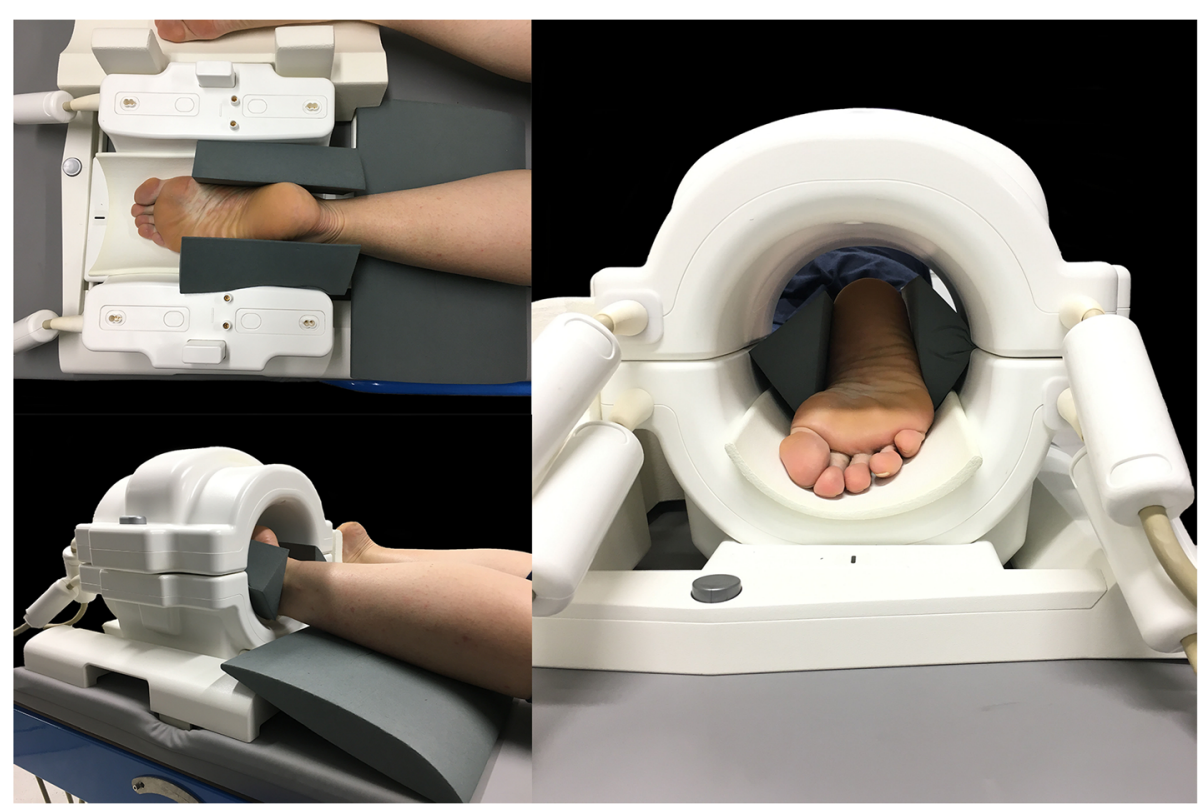

Fig. 1 Participant position for $7 \mathrm{~T}$ and $3 \mathrm{~T}$ scanners

Regions of interest were manually contoured on each slice for each muscle (abductor hallucis, adductor hallucis, flexor digitorum brevis, quadratus plantae, abductor digiti minimi, flexor hallucis brevis, flexor digiti minimi, lumbricals, and plantar and dorsal interossei). Muscle

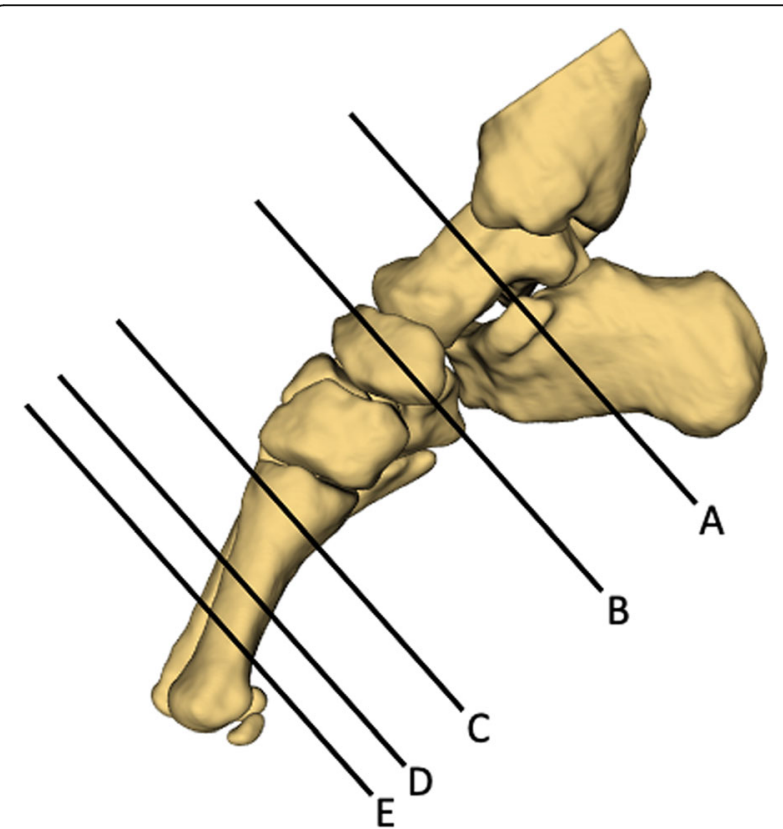

Fig. 2 Image locations: (A) the level of the sustentaculum tali, (B) immediately distal to the talo-navicular joint, $(C)$ immediately distal to the first tarso-metatarsal joints, (D) mid-shaft of the first metatarsal, and (E) distal shaft of the first metatarsal (prior to the metatarsal head) volumes and the percentage of muscle fat infiltration (muscle fat infiltration \% = Fat $/($ Fat + Water) $\mathrm{x} 100)$ were calculated from the segmented images for each muscle using established methods [37, 38]. For illustrative purposes only (viewing the three-dimensional reconstruction), the bones of the leg and foot were also segmented on images.

\section{Results}

Figure 3 presents the coronal plane images at each of the five locations for the $3 \mathrm{~T}$ and $7 \mathrm{~T}$ sequences. For reference, Fig. 3 also displays the region of interest (segmentation) for each muscle on the 7T images. As demonstrated, 7T produced higher image resolution, which enhanced the visualisation of individual muscle borders for manual segmentation. At the rearfoot (Fig. 3a), the abductor hallucis was easily visualised on both $3 \mathrm{~T}$ and $7 \mathrm{~T}$ images, but $7 \mathrm{~T}$ was required to visualise muscle borders between quadratus plantae and abductor digiti minimi, as well as between abductor digiti minimi and flexor digitorum brevis. At the midfoot (Fig. 3b), although individual muscle borders were enhanced on 7T images, muscle borders of extensor digitorum brevis, abductor hallucis, flexor digitorum brevis, quadratus plantae, abductor digiti minimi were visible on both $3 \mathrm{~T}$ and $7 \mathrm{~T}$ images. At the forefoot (Fig. 3c, d and e), the enhanced visualisation of individual muscle borders on $7 \mathrm{~T}$ was required to distinguish flexor digiti minimi from abductor digiti minimi, adductor hallucis from flexor hallucis brevis, and abductor hallucis from flexor hallucis brevis. 7T images also provided sufficient resolution to 


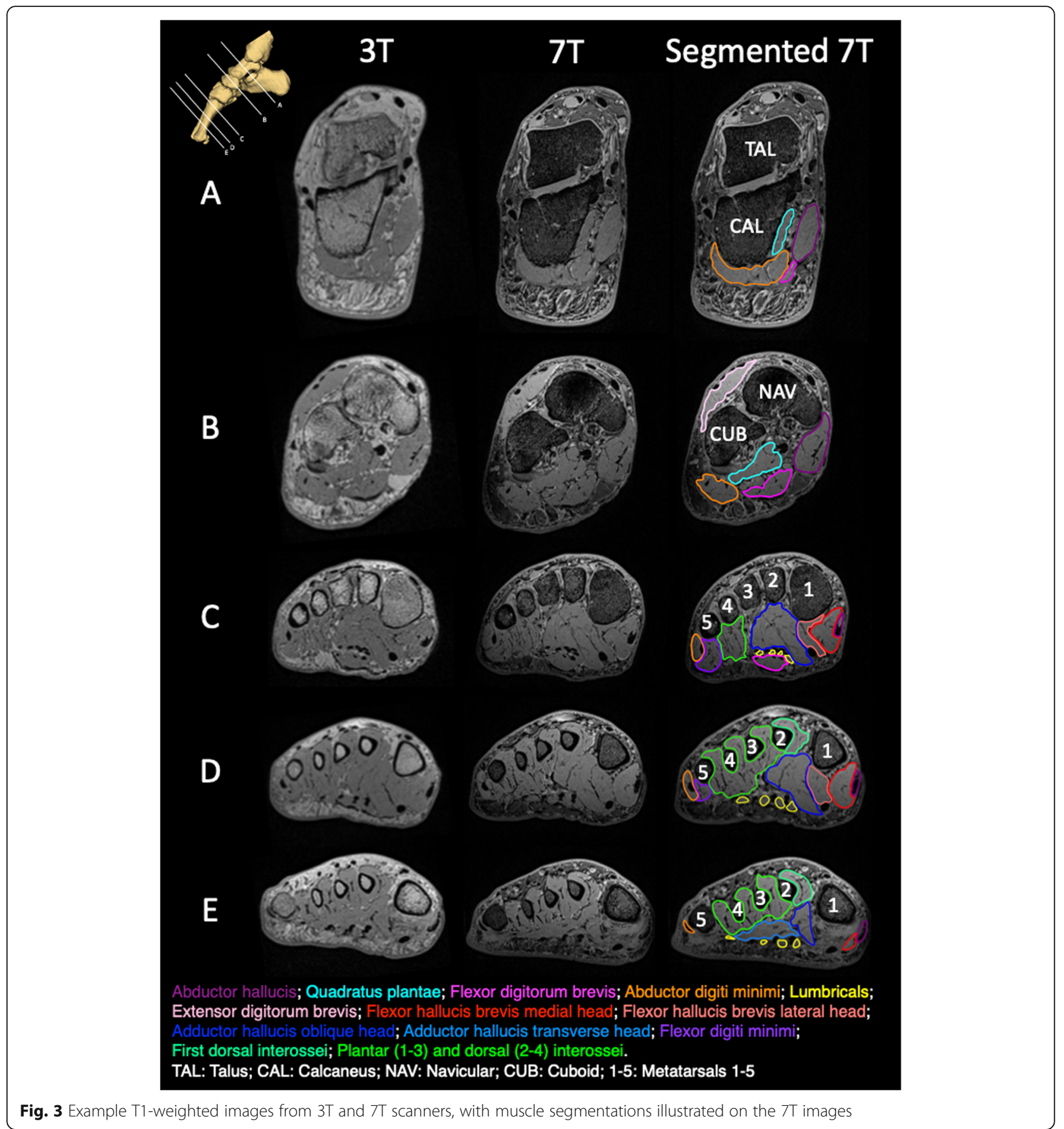

visualise and individually segment discrete portions of some muscles, for example, the transverse and oblique portions of the adductor hallucis (Fig. 3E), as well as the medial and lateral portions of the flexor hallucis brevis (Fig. 3c, d). The muscle border of the first dorsal interossei was visible on 7T and could be segmented individually, however differentiation of the other individual plantar and dorsal interossei was challenging, even on
7T images. Consequently, these muscles were segmented as a group (Fig. 3c, d, e).

Figure 4 illustrates fat and water images, in the coronal plane, from the same five anatomical locations (Fig. 2). For reference, the in-phase image is also included with the region of interest (segmentation) displayed for each muscle. Measurements of muscle volume and percentage muscle fat infiltration are 


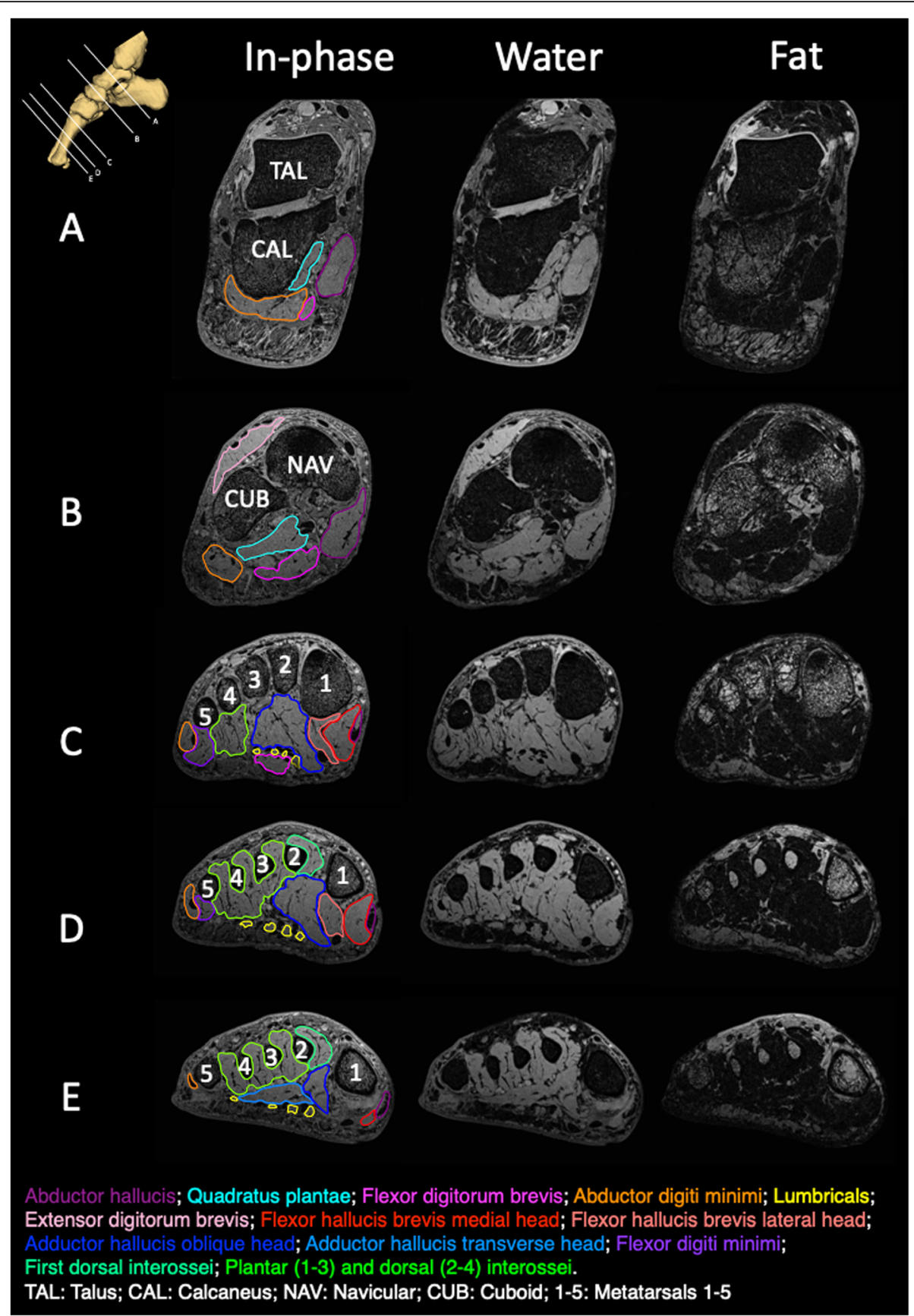

Fig. 4 Example fat-water images from 7T scanner, with muscle segmentations illustrated on the in-phase images

provided in Table 2. Muscle volume ranged from $1.5 \mathrm{~cm}^{3}$ (lumbricals) to $19.8 \mathrm{~cm}^{3}$ (dorsal and plantar interossei) and percentage muscle fat infiltration ranged from 9.2\% (extensor digitorum brevis) to $15.0 \%$ (abductor digiti minimi). Figure 5 displays a three-dimensional reconstruction of the intrinsic foot muscles from 3D Slicer.

\section{Discussion}

Ultra-high-field (7T) MRI enabled the establishment of quantitative methods to provide more detailed measures of intrinsic foot muscle morphology (volume) and composition (percentage muscle fat infiltration) than has been possible with previous technology. In this proof-ofconcept study, we compared images acquired on 7T and 
Table 2 Muscle volume and percentage muscle fat infiltration (MFI)

\begin{tabular}{|c|c|c|}
\hline & Volume $\left(\mathrm{cm}^{3}\right)$ & $\% \mathrm{MFI}$ \\
\hline Abductor hallucis & 17.4 & 11.3 \\
\hline Quadratus plantae & 13.9 & 12.6 \\
\hline Flexor digitorum brevis & 10.2 & 12.4 \\
\hline Abductor digiti minimi & 17.3 & 15.0 \\
\hline \multicolumn{3}{|l|}{ Flexor hallucis brevis } \\
\hline Medial head & 7.8 & 10.8 \\
\hline Lateral head & 5.9 & 11.3 \\
\hline \multicolumn{3}{|l|}{ Adductor hallucis } \\
\hline Oblique head & 17.8 & 11.0 \\
\hline Transverse head & 1.8 & 14.0 \\
\hline Flexor digitorum brevis & 4.4 & 13.5 \\
\hline Lumbricals & 1.5 & 12.2 \\
\hline \multicolumn{3}{|l|}{ Interosseous } \\
\hline First dorsal interosseous & 4.0 & 13.1 \\
\hline Plantar interosseous (1-3) and dorsal interosseous (2-4) & 19.8 & 12.8 \\
\hline Extensor digitorum brevis & 7.7 & 9.2 \\
\hline
\end{tabular}

3T scanners, with similar acquisition times, in a single participant on the same day. As 7T MRI has double the signal-to-noise ratio of $3 \mathrm{~T}$, the image contrast was, as expected, superior at $7 \mathrm{~T}$ scanning. The higher resolution was required to enable clear visualisation of anatomical borders for segmentation of individual muscles, particularly in the forefoot. Our observation is supported by previous reports from several authors that segmenting individual intrinsic foot muscles was challenging at lower field strength due to lower image quality [12, 29,

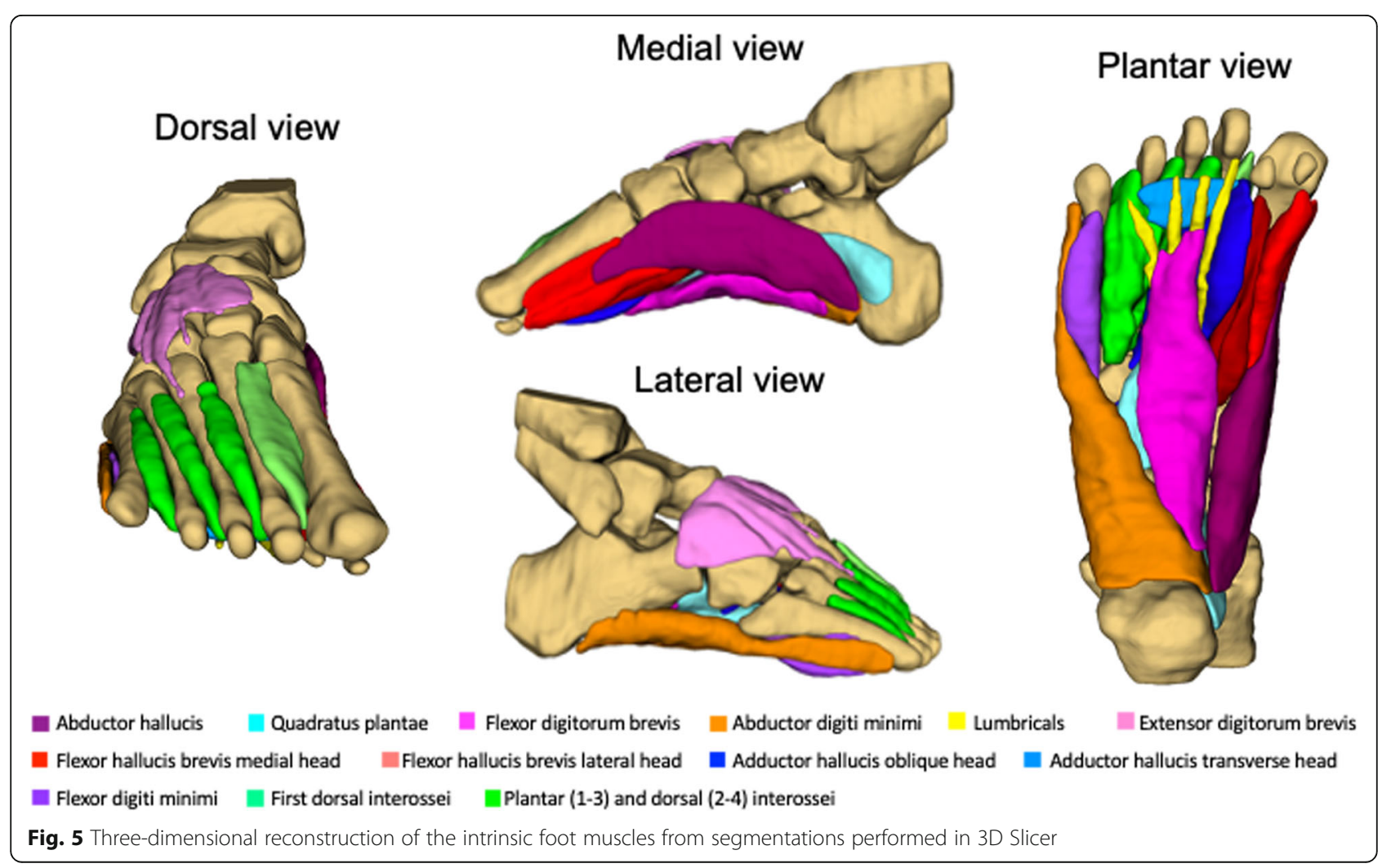


32], as well as the wider literature on morphological musculoskeletal imaging that reports increased spatial resolution at 7T [39].

Although the resolution was lower for the images at $3 \mathrm{~T}$ than $7 \mathrm{~T}$, the lower resolution might be sufficient to enable the segmentation and measurement of crosssectional area of some muscles at some locations. For example, abductor hallucis at the rearfoot and midfoot, and extensor digitorum brevis, flexor digitorum brevis, quadratus plantae and abductor digiti minimi at the midfoot, were visible on the $3 \mathrm{~T}$ images. Previous investigations have reported measurements of cross-sectional area of these muscles of the rearfoot using lower field strengths $(1.5 \mathrm{~T})[20,31]$. However, our findings, and reports from others (using lower magnetic field strengths) $[12,29,32,33]$ of the difficulty to clearly visualise borders of the smaller muscles in the forefoot, draws into question the clinical meaningfulness of individual muscle volumes previously reported from 3T or lower field scanners $[24,25,27,28,30]$. For future studies using magnetic field strengths of $3 \mathrm{~T}$ or less, we caution the segmentation of individual intrinsic foot muscles in the forefoot, and recommend that 7T MRI is used to ensure segmentation precision in that regard. Alternatively, muscle volumes could be reported for muscle groups (e.g. abductor hallucis together with flexor hallucis brevis, abductor digiti minimi together with flexor digiti minimi) [16, 32], or the intrinsic foot muscles as a whole $[7,8,13,14,17-19,26]$, similar to that reported by previous studies.

In this report of a new method, we follow suggestions from the earlier papers in this series [34, 35] towards quantification of muscle fat infiltration for individual intrinsic foot muscles. Using semi-quantitative methods, previous MRI studies at the foot have reported low fatty infiltration in healthy participants $[11,16,20]$. One study that used a quantitative method [13, 14], reported that $22.7 \%$ of total intrinsic foot muscle volume was intramuscular adipose tissue (muscle fat infiltration) in 12 healthy adults (mean age 57 years). This was evaluated for the intrinsic foot muscles as a group, not individual muscles. At other areas of the body, muscle fat infiltration has been observed to differ between individual muscles, within a muscle group, in patient populations [40, 41]. Considering different pain, injury and disease conditions of the foot may affect individual muscles differently, individual muscle segmentation would provide more specific information. We recommend that future studies perform individual muscle segmentations when evaluating muscle fat infiltration, however we recognise this may be limited by availability of ultra-high-field MRI (e.g. 7T).

Although the benefits obtained from 7T compared to 3T for musculoskeletal imaging, such as faster scan time (by a mean factor of 2) and increased spatial resolution ( 2 to 4-fold), have been reported [39], the availability of ultra-high-field MRI is a consideration for research or clinical utility. Availability may be influenced by several factors including cost and patient safety. In 2015 the estimated cost to install a 7T system in the US was approximately $\$ 10$ million, although cost is expected to go down with widespread adoption of systems by institutions/clinics [42]. The field of ultra-high-field imaging is rapidly evolving with growth from approximately 40 scanners worldwide in 2014 [43] to over 80 scanners worldwide in 2019 [44]. This evolution also includes transition from use primarily for scientific and medical research to the first clinical use at the Mayo Clinic Rochester in 2017, as well as the number of scanners available in the marketplace. User costs are also a consideration for researchers and patients. To provide context, at our institution $7 \mathrm{~T}$ scan costs are $30-40 \%$ higher than $3 \mathrm{~T}$ for the same scanning time period (e.g. $30 \mathrm{mi}$ nute scan). In terms of patient safety, relatively few biomedical implants have undergone proper testing at $7 \mathrm{~T}$ and therefore to mitigate risks related to increased forces on metallic implants and the unpredictable radiofrequency-induced tissue heating with ultra-highfield strengths, a significant portion of research participants/patients may be precluded from currently undergoing $7 \mathrm{~T}$ imaging [45].

Operational parameters can influence the quality of images that are acquired from MRI. As is evident in Table 1, a wide range of parameters have been used to evaluate intrinsic foot muscle morphology in previous work and this has been reported with variation in detail. To enable comparisons between foot imaging studies in future, standardised reporting of parameters is required. We propose reporting of the following operation parameters: field strength (e.g. 7-Tesla); sequence type (e.g. 2point DIXON (3D fast-field echo T1) whole foot); repetition time (e.g. TR $11 \mathrm{~ms}$ ); echo time (e.g. TE $3.06 \mathrm{~ms}$ and $5.61 \mathrm{~ms}$ ); flip angle (e.g. $3^{\circ}$ ); field of view (e.g. FOV $111 \times 223 \mathrm{~mm}^{2}$ ); acquired voxel dimensions (e.g. $0.38 \times$ $0.38 \mathrm{~mm}^{2}$ ); reconstructed voxel dimensions (e.g. $0.38 \times$ $0.38 \mathrm{~mm}^{2}$ ); bandwidth (e.g. $434 \mathrm{~Hz} / \mathrm{Px}$ ); acquisition time (e.g. TA $11 \mathrm{~min} 49 \mathrm{~s}$ ); slice thickness (e.g. $0.38 \mathrm{~mm}$ ) and number of sliced (e.g. 640 slices). Additionally, to improve transparency and reproducibility, image measurement methods should be described in detail, with the strong recommendation to include figures to illustrate muscle segmentation.

We report images of the intrinsic foot muscles acquired using 3T and 7T with one healthy individual, towards establishing the feasibility of quantifying intrinsic foot muscle structure and composition using higherfield (7T) MRI. It is our hope that this foundational effort can facilitate future studies using these methods, 
both in healthy individuals and in those with lower limb pain, injury or disease, in order to: (i) enhance understanding of structure and compositional changes in the intrinsic foot muscles across different populations and (ii) generate a body of literature that can be pooled and further scrutinised in systematic reviews with metaanalyses. Although our data illustrates the difference in image quality between $7 \mathrm{~T}$ and $3 \mathrm{~T}$ scanners, further research is required to assess the influence of field strength on measurement properties, such as the repeatability of measurements, an aspect that is lacking from the existing literature (see Table 1). Validity of measures should be considered by comparison with anatomical atlases from cadaveric tissue; as reported in the previous methods papers $[34,35]$. As manual segmentation of the individual intrinsic foot muscles is time consuming (approximately 6 hours per foot for our data) and raterdependent, development of automated methods may improve the efficiency and objectivity of muscle measures. Deep learning convolutional neural network models for muscle segmentation and automatic muscle fat infiltration calculation using fat-water images have demonstrated high test reliability and accuracy for muscles in other body regions [46]. This is an area that should be explored in future studies, as it may improve the ability to quantify and monitor foot muscle structure and composition in individuals with lower limb pain, injury or disease; saving time from arduous manual segmentation techniques, and increase likelihood of clinical implementation.

\section{Conclusions}

We have used ultra-high-field (7T) MRI to establish a method to quantify muscle morphology and composition of individual intrinsic foot muscles. This method can be used in future studies to better understand intrinsic foot muscle morphology and composition in healthy individuals, as well as those with lower limb disorders. Following on from papers at the cervical and lumbar spine [34, 35], we also emphasise that improved reporting of image acquisition parameters and image measurement procedures is required to assist consistency and allow accurate comparison between studies.

\section{Abbreviations}

MRI: Magnetic resonance imaging; T: Tesla; TE: Echo time; TR: Repetition time; US: Ultrasound imaging

\section{Acknowledgements}

The authors acknowledge the facilities, and the scientific and technical assistance of the National Imaging Facility at the Centre for Advanced Imaging, University of Queensland.

\section{Authors' contributions}

NJC, MMFS and JME conceived the study, while AA, KAW, MAH, BV and PWH each made substantial contributions to its design. NJC, MMFS and JME have been involved in drafting the manuscript. All authors have been involved in revising it critically for important intellectual content and each given final approval of the version to be published. All authors have read and approved the final manuscript.

\section{Funding}

MMFS is supported by a University of Queensland Development Fellowship (UQFEL 1832429). KAW is supported by grants from the National Institute of Neurological Disorders and Stroke (K23NS104211 and L30NS108301). PWH is supported by the National Health and Medical Research Council of Australia (NHMRC: APP1091302 and APP1102905). The content is solely the responsibility of the authors and does not necessarily represent the official views of the National Institutes of Health.

Availability of data and materials

All of the data supporting the findings are contained within the manuscript.

Ethics approval and consent to participate

Ethical approval was provided by The University of Queensland Human Research Ethics Committee (\#2018001150). The participant provided informed written consent.

\section{Consent for publication}

All MRIs were derived from the same informed and consenting adult participant.

\section{Competing interests}

The authors declare that they have no competing interests.

\section{Author details}

${ }^{1}$ School of Health and Rehabilitation Sciences, The University of Queensland, 4072 Brisbane, QLD, Australia. ${ }^{2}$ Faculty of Medicine and Health, The Kolling Research Institute, The University of Sydney, the Northern Sydney Local Health District, 2006 Sydney, New South Wales, Australia. ${ }^{3}$ Department of Physical Therapy and Human Movement Sciences, Northwestern University, Chicago, IL, USA. ${ }^{4}$ Centre for Advanced Imaging, The University of Queensland, 4072 Brisbane, QLD, Australia. ${ }^{5}$ Systems Neuroscience and Pain Lab, Division of Pain Medicine, Department of Anesthesiology, Perioperative and Pain Medicine, Stanford University School of Medicine, Palo Alto, CA, USA. ${ }^{6}$ La Trobe Sport and Exercise Medicine Research Centre, School of Allied Health, Human Services and Sport, College of Science, Health and Engineering, La Trobe University, 3086 Melbourne, Australia.

Received: 4 August 2020 Accepted: 26 December 2020

Published online: 21 January 2021

\section{References}

1. Kelly LA, Farris DJ, Cresswell AG, Lichtwark GA. Intrinsic foot muscles contribute to elastic energy storage and return in the human foot. J Applied Physiol. 2019;126:231-8.

2. Farris DJ, Kelly LA, Cresswell AG, Lichtwark GA. The functional importance of human foot muscles for bipedal locomotion. P Natl Acad Sci USA. 2019; 116(5):1645-50.

3. Riddick R, Farris DJ, Kelly LA. The foot is more than a spring: human foot muscles perform work to adapt to the energetic requirements of locomotion. J R Soc Interface. 2019;16(150):20180680.

4. Soysa A, Hiller C, Refshauge K, Burns J. Importance and challenges of measuring intrinsic foot muscle strength. J Foot Ankle Res. 2012:5(1):29.

5. Lieber RL, Friden J. Clinical significance of skeletal muscle architecture. Clin Orthop Relat R. 2001;383:140-51.

6. McGregor RA, Cameron-Smith D, Poppitt SD. It is not just muscle mass: a review of muscle quality, composition and metabolism during ageing as determinants of muscle function and mobility in later life. Longev Healthspan. 2014;3:9.

7. Andersen $\mathrm{H}, \mathrm{Gjerstad}$ MD, Jakobsen J. Atrophy of foot muscles: a measure of diabetic neuropathy. Diabetes Care. 2004;27(10):2382-5.

8. Andreassen CS, Jakobsen J, Ringgaard S, Ejskjaer N, Andersen H. Accelerated atrophy of lower leg and foot muscles-a follow-up study of long-term diabetic polyneuropathy using magnetic resonance imaging (MRI). Diabetologia. 2009;52(6):1182-91. 
9. Brash PD, Foster J, Vennart W, Anthony P, Tooke JE. Magnetic resonance imaging techniques demonstrate soft tissue damage in the diabetic foot. Diabet Med. 1999;16(1):55-61.

10. Bus SA, Maas M, Lindeboom R. Reproducibility of foot structure measurements in neuropathic diabetic patients using magnetic resonance imaging. J Magn Reson Imaging. 2006;24(1):25-32.

11. Bus SA, Maas M, Michels RPJ, Levi M. Role of intrinsic muscle atrophy in the etiology of claw toe deformity in diabetic neuropathy may not be as straightforward as widely believed. Diabetes Care. 2009;32(6):1063-7.

12. Bus SA, Yang QX, Wang JH, Smith MB, Wunderlich R, Cavanagh PR. Intrinsic muscle atrophy and toe deformity in the diabetic neuropathic foot: a magnetic resonance imaging study. Diabetes Care. 2002;25(8):1444-50.

13. Cheuy VA, Commean PK, Hastings MK, Mueller MJ. Reliability and validity of a MR-based volumetric analysis of the intrinsic foot muscles. J Magn Reson Imaging. 2013;38(5):1083-93.

14. Cheuy VA, Hastings MK, Commean PK, Ward SR, Mueller MJ. Intrinsic foot muscle deterioration is associated with metatarsophalangeal joint angle in people with diabetes and neuropathy. Clin Biomech. 2013;28(9-10):1055-60.

15. Greenman RL, Khaodhiar L, Lima C, Dinh T, Giurini JM, Veves A. Foot small muscle atrophy is present before the detection of clinical neuropathy. Diabetes Care. 2005;28(6):1425-30.

16. Lin YC, Wu J, Baltzis D, Veves A, Greenman R. MRI assessment of regional differences in phosphorus-31 metabolism and morphological abnormalities of the foot muscles in diabetes. J Magn Reson Imaging. 2016;44(5):1132-42.

17. Severinsen K, Obel A, Jakobsen J, Andersen H. Atrophy of foot muscles in diabetic patients can be detected with ultrasonography. Diabetes Care. 2007;30(12):3053-7.

18. Chang R, Kent-Braun JA, Hamill J. Use of MRI for volume estimation of tibialis posterior and plantar intrinsic foot muscles in healthy and chronic plantar fasciitis limbs. Clin Biomech. 2012;27(5):500-5.

19. Cheung RT, Sze LK, Mok NW, Ng GY. Intrinsic foot muscle volume in experienced runners with and without chronic plantar fasciitis. J Sci Med Sport. 2016;19(9):713-5.

20. Schmid DT, Hodler J, Mengiardi B, Pfirrmann CW, Espinosa N, Zanetti M. Fatty muscle atrophy: prevalence in the hindfoot muscles on MR images of asymptomatic volunteers and patients with foot pain. Radiology. 2009; 253(1):160-6.

21. Recht MP, Grooff P, Ilaslan H, Recht HS, Sferra J, Donley BG. Selective atrophy of the abductor digiti quinti: an MRI study. Am J Roentgenol. 2007; 189(3):W123-W7.

22. Pelayo-Negro AL, Gallardo E, Garcia A, Sanchez-Juan P, Infante J, Berciano J. Evolution of Charcot-Marie-Tooth disease type 1A duplication: a 2-year clinico-electrophysiological and lower-limb muscle MRI longitudinal study. J Neurol. 2014:261(4):675-85.

23. Gallardo E, Garca A, Combarros O, Berciano J. CharcotMarieTooth disease type 1A duplication: spectrum of clinical and magnetic resonance imaging features in leg and foot muscles. Brain. 2006;129(2):426-37.

24. Feger MA, Donovan L, Herb CC, Handsfield GG, Blemker SS, Hart JM, et al. Impairment-Based Rehabilitation Increases Lower Leg Muscle Volumes and Strength in Chronic Ankle Instability Patients: A Preliminary Study. J Sport Rehabil. 2019;28(5):450-8.

25. Feger MA, Snell S, Handsfield GG, Blemker SS, Wombacher E, Fry R, et al. Diminished Foot and Ankle Muscle Volumes in Young Adults With Chronic Ankle Instability. Orthop J Sports Med. 2016;4(6):2325967116653719-.

26. Chen TL-W, Sze LKY, Davis IS, Cheung RTH. Effects of training in minimalist shoes on the intrinsic and extrinsic foot muscle volume. Clin Biomech. 2016; 36:8-13.

27. Miller EE, Whitcome KK, Lieberman DE, Norton HL, Dyer RE. The effect of minimal shoes on arch structure and intrinsic foot muscle strength. J Sport Health Sci. 2014;3(2):74-85.

28. Taddei UT, Matias AB, Ribeiro FIA, Inoue RS, Bus SA, Sacco ICN. Effects of a therapeutic foot exercise program on injury incidence, foot functionality and biomechanics in long-distance runners: Feasibility study for a randomized controlled trial. Phys Ther Sport. 2018:34:216-26.

29. Gooding TM, Feger MA, Hart JM, Hertel J. Intrinsic Foot Muscle Activation During Specific Exercises: A T2 Time Magnetic Resonance Imaging Study. J Athl Train. 2016;51(8):644-50.

30. Taddei UT, Matias AB, Ribeiro FIA, Bus SA, Sacco ICN. Effects of a foot strengthening program on foot muscle morphology and running mechanics: A proof-of-concept, single-blind randomized controlled trial. Phys Ther Sport. 2020;42:107-15.
31. Savnik A, Amris K, Røgind H, Prip K, Danneskiold-Samsøe B, Bojsen-Møller F, et al. MRI of the plantar structures of the foot after falanga torture. Eur Radiol. 2000;10(10):1655-9.

32. Green SM, Briggs PJ. Flexion strength of the toes in the normal foot. An evaluation using magnetic resonance imaging. Foot. 2013;23(4):115-9.

33. Kurihara T, Yamauchi J, Otsuka M, Tottori N, Hashimoto T, Isaka T. Maximum toe flexor muscle strength and quantitative analysis of human plantar intrinsic and extrinsic muscles by a magnetic resonance imaging technique. J Foot Ankle Res. 2014;7:26-.

34. Crawford RJ, Cornwall J, Abbott R, Elliott JM. Manually defining regions of interest when quantifying paravertebral muscles fatty infiltration from axial magnetic resonance imaging: a proposed method for the lumbar spine with anatomical cross-reference. BMC Musculoskel Dis. 2017;18(1):25.

35. Elliott JM, Cornwall J, Kennedy E, Abbott R, Crawford RJ. Towards defining muscular regions of interest from axial magnetic resonance imaging with anatomical cross-reference: part II - cervical spine musculature. BMC Musculoskel Dis. 2018;19(1):171-

36. Fedorov A, Beichel R, Kalpathy-Cramer J, Finet J, Fillion-Robin J-C, Pujol S, et al. 3D Slicer as an image computing platform for the Quantitative Imaging Network. Magn Reson Imaging. 2012;30(9):1323-41.

37. Khanna R, Saltzman MD, Elliott JM, Hoggarth MA, Marra GM, Omar I, et al. Development of 3D method to assess intramuscular spatial distribution of fat infiltration in patients with rotator cuff tear: reliability and concurrent validity.(Report). BMC Musculoskel Dis. 2019;20(1):295-5.

38. Crawford JR, Volken CT, Ni Mhuiris M, Bow AC, Elliott AJ, Hoggarth AM, et al. Geography of Lumbar Paravertebral Muscle Fatty Infiltration: The Influence of Demographics, Low Back Pain, and Disability. Spine. 2019; 44(18):1294-302.

39. Juras V, Mlynarik V, Szomolanyi P, Valkovič L, Trattnig S. Magnetic Resonance Imaging of the Musculoskeletal System at 7T: Morphological Imaging and Beyond. Top Magn Reson Imaging. 2019;28(3):125-35.

40. Uthaikhup S, Assapun J, Kothan S, Watcharasaksilp K, Elliott JM. Structural changes of the cervical muscles in elder women with cervicogenic headache. Musculoskelet Sci Pract. 2017;29:1-6.

41. Elliott J, Jull G, Noteboom JT, Darnell R, Galloway G, Gibbon WW. Fatty infiltration in the cervical extensor muscles in persistent whiplashassociated disorders: a magnetic resonance imaging analysis. Spine. 2006;31(22):E847-E855.

42. Balchandani P, Naidich TP. Ultra-High-Field MR, Neuroimaging. Am J Neuroradiol. 2014;36(7):1204-15.

43. Regatte RR. Why Buy an Expensive (\$7 Million) 7T MRI System for Biomedical Research? J Magn Reson Imaging. 2014;40(2):280-2.

44. Barisano G, Sepehrband F, Ma S, Jann K, Cabeen R, Wang DJ, et al. Clinical 7 T MRI: Are we there yet? A review about magnetic resonance imaging at ultra-high field. British J Radiol. 2019;92(1094):20180492.

45. Hoff MN, McKinney At, Shellock FG, Rassner U, Gilk T, Watson RE Jr, et al. Safety Considerations of 7-T MRI in Clinical Practice. Radiology. 2019;292(3): 509-18.

46. Weber KA, Smith AC, Wasielewski M, Eghtesad K, Upadhyayula PA, Wintermark M, et al. Deep Learning Convolutional Neural Networks for the Automatic Quantification of Muscle Fat Infiltration Following Whiplash Injury. Sci Rep. 2019;9(1):7973.

\section{Publisher's Note}

Springer Nature remains neutral with regard to jurisdictional claims in published maps and institutional affiliations.

\section{Ready to submit your research? Choose BMC and benefit from:}

- fast, convenient online submission

- thorough peer review by experienced researchers in your field

- rapid publication on acceptance

- support for research data, including large and complex data types

- gold Open Access which fosters wider collaboration and increased citations

- maximum visibility for your research: over $100 \mathrm{M}$ website views per year

At $\mathrm{BMC}$, research is always in progress.

Learn more biomedcentral.com/submission 